\title{
Mda-7/IL-24 induces the differentiation of $B$ cell lymphoma via activation of the $P 38$ mitogen activated protein kinase signaling pathway
}

\author{
MING MA ${ }^{1 *}$, XINGXIAO YANG ${ }^{2 *}$, LIANMEI ZHAO ${ }^{3}$, LIHUA LIU $^{4}$, \\ CONG ZHANG $^{3}$, XUEXIAO WANG ${ }^{4}$ and BAOEN SHAN ${ }^{3}$ \\ ${ }^{1}$ Clinical Laboratory; ${ }^{2}$ Department of Infection Management; ${ }^{3}$ Research Center; ${ }^{4}$ Department of Biotherapy, \\ The Fourth Hospital of Hebei Medical University, Shijiazhuang, Hebei 050011, P.R. China
}

Received October 8, 2016; Accepted June 1, 2017

DOI: $10.3892 / \mathrm{mmr} .2017 .7306$

\begin{abstract}
Interleukin 24 (IL-24) is a unique cytokine encoded by the melanoma differentiation associated gene-7 (Mda-7), and was first discovered inhuman melanoma cells. Exogenous Mda-7/IL-24 has been shown to inhibit the proliferation and invasion of a broad spectrum of human cancer cells, but its effect on the differentiation of B cell lymphoma is not yet clear. To the best of our knowledge, the present study demonstrated for the first time that overexpressing Mda-7/IL-24 can induce differentiation in human B cell lymphomacells, and the underlying mechanism was investigated. The proliferation of stable Mda-7/IL-24 overexpressing Raji and Daudi cells was assessed by the MTS method. The immunophenotype, apoptosis level and cell cycle distribution of Raji and Daudi cells were analyzed by flow cytometry. The expression of PR domain zinc finger protein 1 (Blimp1) and B-cell lymphoma 6 (Bcl6) were analyzed by western blotting. Additionally, western blotting assay was also performed to study the effect of Mda-7/IL-24 on the activity of the P38 mitogen activated protein kinase (MAPK) signaling pathway in Raji and Daudi cells. Proliferation of Raji and Daudi cells overexpressing Mda-7/IL-24 was inhibited significantly, compared with those of parent cells and cells transfected with the empty vector alone. Apoptosis was not involved in the proliferation inhibition,
\end{abstract}

Correspondence to: Professor Baoen Shan, Research Center, The Fourth Hospital of Hebei Medical University, 12 Jiankang Road, Shijiazhuang, Hebei 050011, P.R. China

E-mail: shanbaoen@sohu.com

*Contributed equally

Abbreviations: Mda-7/IL-24, melanoma differentiation associated gene7/interleukin-24; FCM, flow cytometry; MAPK, mitogen activated protein kinase

Key words: Mda-7/IL-24, B cell lymphoma, differentiation induction, p38 MAPK while the cell cycle was arrested in G1 phase in the Raji and Daudi cells overexpressing Mda-7/IL-24. Overexpressing Mda-7/IL-24 resulted in a significantly decreased expression of cluster of differentiation (CD)10, and increased expression of CD45 and CD138 in the cell surface of Raji and Daudi cells. The expression of Blimp1 was upregulated, while the levels of Bcl6 protein was downregulated, in Raji and Daudi cells overexpressing Mda-7/IL-24. Furthermore, the activities of the P38 MAPK signaling pathway in lymphoma cells were upregulated. These results indicated that Mda-7/IL-24 could induce terminal differentiation of B lymphoma cells by regulating the expression of Blimp1 and Bcl6 via altering the P38 MAPK signaling pathway, suggesting that Mda-7/IL-24 may therefore be a potential differentiation therapeutic agent to be applied in clinical treatment of B cell lymphoma.

\section{Introduction}

B cell lymphoma is a clinical hematopoietic malignancy originating from the lymphoid system and is the most common subtype of non-Hodgkin's lymphoma, with high mortality worldwide (1). The prevalence of B cell lymphoma and its poor prognosis following chemotherapy indicates requirement to identify alternativestrategies for its treatment (2). A novel approach for tumor treatment is to use agents that inhibit growth of tumor cells by inducing terminal differentiation, a strategytermed 'differentiation therapy' (3). Hematopoietic malignancy including B cell lymphoma are characterized by a blockage in differentiation. Since the establishment of all-trans retinoic acid as a classical differentiation inducer to treat acute promyelocytic leukemia (AML-M3), AML-M3 has become a curable subtype of acute myeloid leukemia (AML) $(3,4)$. However, agents such as B cell lymphoma differentiation inducer, are extremely less developed. As differentiation therapy can avoid many disadvantages of chemotherapy, there is an urgent requirement to design more effective strategies to induce differentiation of B cell lymphoma. Meanwhile, investigation of the underlying mechanism associated with lymphoma cell differentiation may provide useful therapeutic targets for identifying specific and safe differentiation therapy to treat lymphoma. 
Interleukin (IL)-24, a unique cytokine that belongs to the IL-10 cytokine family, exhibits antitumor activity against various human malignant tumor cells. IL-24 is encoded by the melanoma differentiation associated gene-7 (Mda-7), and was first identified in human melanoma cells (5). Mda-7/IL-24 expression is lost in a variety of cancers, including leukemia and lymphoma (6,7). Although its physiological role is poorly understood, overexpression of Mda-7/IL-24 was reported to result in growth inhibition, reversal of malignant phenotype, cell cycle arrest, induction of apoptosis and terminal differentiation in a broad spectrum of malignant tumors, but to have non-toxic side effects on healthy human cells (7-13). Previously, Mda-7/IL-24 was also observed to induce monocytic differentiation of human HL60 (promyelocytic leukemia) and U937 (promonocytic leukemia) cell lines (3). This ability of Mda-7/IL-24 to induce differentiation in AML cells led to the hypothesis that overexpression of Mda-7/IL-24 may serve a similar role in B cell lymphoma cells.

To verify this hypothesis, the present study investigated the effect of overexpressing Mda-7/IL-24 on the growth, invasion, migration and differentiation of B cell lymphoma cells in vitro, with the goal of clarifying the potential molecular mechanism of Mda-7/IL-24. To the best of our knowledge, we have demonstrated for the first time that overexpression of Mda-7/IL-24 can induce terminal differentiation in human B cell lymphoma cells in vitro. Raji and Daudi cells transfected with Mda-7/IL-24 demonstrated significant growth suppression. The number of cells in G0/G1 phase was increased, and cells in $\mathrm{S}$ phase were decreased in lymphoma cells overexpressing Mda-7/IL-24. Furthermore, the expression of immature B lymphocyte surface marker cluster of differentiation (CD) 10 was significantly decreased, while CD45 and CD138 were increased in Raji and Daudi cells overexpressing Mda-7/IL-24. Additionally, the expression of C-myc, N-myc and B-cell lymphoma 6 (Bcl6) was significantly downregulated, whereas PR domain zinc finger protein 1 (Blimp1) expression was upregulated, in lymphoma cells overexpressing Mda-7/IL-24.

These results suggested that overexpression of Mda-7/IL-24 can induce Raji and Daudi cell differentiation, and thus may represent a potential clinical differentiation therapeutic agent to be applied in patients with lymphoma.

\section{Materials and methods}

Cell lines and reagents. Raji and Daudi human B lymphoma cells were supplied by the research center of the Fourth Hospital of Hebei Medical University (Hebei, China). RPMI-1640 and 3-(4,5-dimethylthiazol-2-yl)-5-(3-carboxym ethoxyphenyl)-2-(4-sulfophenyl)-2H-tetazolium (MTS) was obtained from Sigma-Aldrich; Merck KGaA (Darmstadt, Germany). Fetal calf serum (FCS) was purchased from Gibco; Thermo Fisher Scientific, Inc. (Waltham, MA, USA). Annexin V-fluorescein isothiocyanate (FITC), a7-aminoactinomycin D (7-AAD) double stain kit, CD10-FITC, CD19-allophycocyanin (APC), CD138-FITC and CD45-APC were all purchased from BD Pharmingen; BD Biosciences (San Jose, CA, USA). The radio-immunoprecipitation assay lysis buffer and bicinchoninic acid protein assay kit were purchased from Beijing Solarbio Science \& Technology
Co., Ltd. (Beijing, China). Rabbit anti-human antibodies against phosphorylated (p)-P38 (cat no. 4631S), Blimp1 (cat no. 9115) and Bcl6 (cat no. 14895S) were all obtained from Cell Signaling Technology, Inc. (Danvers, MA, USA). Rabbit anti-human antibodies against total P38 MAPK (cat no. 66234-1-Ig) and matrix metalloproteinase (MMP)2 (cat no. 10373-2-Ab) were purchased from Proteintech Group, Inc. (Rosemont, IL, USA). Rabbit anti-human antibodies against c-myc (cat no. 189790-2), N-myc (cat no. 153733-2), Mda-7/IL-24 (cat no. GR/52203-2) and MMP9 (cat no. 573144) were obtained from Abcam (Cambridge, MA, USA). Rabbit anti-human antibody against $\beta$-actin (cat no. AP0060) was supplied by Bioworld Technology, Inc. (Louis park, MN, USA). The horseradish peroxidase-conjugated goat anti-rabbit secondary antibody (cat no. 29527) was purchased from Rockland (Limerick, PA, USA). Lipofectamine $^{\mathrm{TM}} 2000$ was purchased from Invitrogen; Thermo Fisher Scientific, Inc. A plasmid encoding the human Mda-7/IL-24 gene (pLenti6.3-MCS-Mda-7/IL-24) was synthesized by Invitrogen; Thermo Fisher Scientific, Inc. A pPACKH1 $^{\mathrm{TM}}$ Lentivector Packaging kit was purchased from System Biosciences (Palo Alto, CA, USA). A P38 MAPK inhibitor (SB203580) was purchased from Sigma-Aldrich; Merck KGaA and dissolved in dimethyl sulfoxide [DMSO; the final concentration of DMSO in medium was $<0.1 \%(\mathrm{v} / \mathrm{v})]$ to yield a $1.0 \mathrm{mg} / \mathrm{ml}$ stock solution.

Transfection. Raji and Daudi cells were stably transfected with the lentiviral vector encoding the human Mda-7/IL-24 gene using Lipofectamine 2000 and a pPACKH1 Lentivector Packaging kit. In brief, 2 days prior to transfection, 293T cells $\left(1 \times 10^{6}\right.$ cells/well) were cultured in 6-well culture plates (Gibco; Thermo Fisher Scientific, Inc.) until they reached 70\% confluency, and then the $293 \mathrm{~T}$ cells were transfected with pLenti6.3-MCS-Mda-7/IL-24 and the lentivirus packaging plasmids (pPACKH1-REV, pPACKH1-GAG and pVSV-G) using Lipofectamine 2000. Raji and Daudi cells were co-cultured with the supernatants of $293 \mathrm{~T}$ cells producing the Mda-7/IL-24 gene linked to a lentiviral vector and selected using a fluorescence activated cell sorting (FACSARIA II; BD Biosciences). The collected cells overexpressing Mda-7/IL-24 were sub-cultivated every 3 days. In addition, Raji and Daudi cells were stably transfected with the lentiviral vector alone as a negative control.

MTS assay. The effect of Mda-7/IL-24 on lymphoma cell viability was determined by MTS assay according to the manufacturer's protocol. Raji and Daudi cells $\left(1 \times 10^{5}\right.$ cells/well) were seeded into 96-well plates with $200 \mu 1$ RPMI-1640 containing 10\% FCS. After incubation for 24, 48,72 or $96 \mathrm{~h}$ at $37^{\circ} \mathrm{C}, 10 \mu \mathrm{l}$ MTS solution $(5 \mathrm{mg} / \mathrm{ml}$ in PBS) was added to the cells, which were incubated for $4 \mathrm{~h}$ again. The absorbance at $492 \mathrm{~nm}$ was measured with a microplate reader (Titertek Multiskan, North Ryde, Australia) to assess cell viability.

Flow cytometry (FCM). To assess the effect of Mda-7/IL-24 on apoptosis, FCM was performed to analyze the Raji and Daudi cells incubated with Annexin V-FITC and 7-AAD double staining. To analyze the cell cycle distribution of 
Raji and Daudi cells, single-cell suspensions of the two lymphoma cell lines $\left(1 \times 10^{6}\right.$ cells/sample) were treated with RNase A and then fixed in $70 \%$ ethanol overnight at $4^{\circ} \mathrm{C}$. After staining with propidium iodide at room temperature for $15 \mathrm{~min}$, the cells were resuspended in ice-cold PBS, and were analyzed using a flow cytometer (FACS Caliber ${ }^{\mathrm{TM}}$, BD Biosciences).

The effect of Mda-7/IL-24 on the immunophenotype of lymphoma cells was also assessed by FCM. Briefly, the single-cell suspensions of Raji and Daudi cells $\left(1 \times 10^{6}\right.$ cells/sample) were incubated with CD10-FITC and CD45-APC (or CD138-FITC and CD19-APC) double staining at room temperature and analyzed by flow cytometry. Data were analyzed with CellQuest Proversion 5.1 software (BD Biosciences) and are expressed as the mean \pm standard deviation of three independent experiments.

Western blotting. The cultured lymphoma cells were lysed in $500 \mu 1$ RIPA lysis buffer (1\% Triton X-100, $150 \mathrm{mM} \mathrm{NaCl}$, $10 \mathrm{mM}$ Tris-HCl at $\mathrm{pH}$ 7.4, 1 mM EDTA, 1 mM EGTA at $\mathrm{pH}$ 8.0, $0.2 \mathrm{mM}$ Na 3 VO 4, $0.2 \mathrm{mM}$ phenylmethyl sulphonyl fluoride and $0.5 \% \mathrm{NP}-40)$. The protein concentration in the lysates was determined by BCA assay. The lysates (40 $\mu \mathrm{g}$ per well of the SDS-PAGE gels) were separated by SDS-PAGE and were transferred onto polyvinylidene difluoride (PVDF) membranes. The PVDF membranes were blocked in PBS with 5\% bovine serum albumin (Gibco; Thermo Fisher Scientific, Inc.) for $2 \mathrm{~h}$ at room temperature, followed by overnight incubation with specific dilutions of the primary antibodies at $4^{\circ} \mathrm{C}$, including antibodies against Mda-7/IL-24 (1:1,000), P38 (1:1,000), p-P38 (1:1,000), MMP2 (1:1,000), MMP9 (1:2,000), Blimp1 (1:1,000), $\operatorname{Bcl6}(1: 1,000), \mathrm{C}$-myc $(1: 2,000), \mathrm{N}$-myc $(1: 1,000)$ and $\beta$-actin $(1: 5,000)$. The PVDF membranes were visualized in the Odyssey infrared imaging system, after incubation with horseradish peroxidase-conjugated goat anti-rabbit secondary antibodies (cat no. 29527; 1:5,000; Rockland). The levels of protein in each sample were calculated as the ratio of the intensity of protein to those of $\beta$-actin, using Odyssey version 3.0 software (LI-COR Biosciences, Lincoln, NE, USA).

Tumor cell invasion and migration assays. Effects of Mda-7/IL-24 on cell invasion and migration were assessed using Transwell assays in a 24-well Transwell chamber (Collaborative Biomedical, Becton Dickinson Labware, Bedford, MA), which was precoated with Matrigel (for invasion assays) or without Matrigel (for migration assays). In brief, Raji and Daudi cells $\left(2 \times 10^{5}\right.$ cells/well) were seeded in the upper chambers and incubated in $500 \mu \mathrm{l}$ RPMI without FBS, while $500 \mu \mathrm{l}$ medium in the lower chamber contained $10 \%$ FBS as chemoattractant. The plates were incubated for $24 \mathrm{~h}$ in a $5 \% \mathrm{CO}_{2}$ humidified incubator at $37^{\circ} \mathrm{C}$. The cells on the lower side of the filters were stained with $0.5 \%$ crystal violetat room temperature and were counted at $\times 200$ magnification in 5 random fields of each filter.

Statistical analysis. Statistical analysis was conducted with SPSS software package version 13.0 (SPSS, Inc., Chicago, IL, USA). All data are presented as the mean \pm standard deviation of at least three independent experiments, and analyzed by one-way analysis of variance followed by Tukey's post hoc test for multiple comparisons. A $\mathrm{P}<0.05$ was considered to indicate a statistically significant difference.

\section{Results}

Mda-7/IL-24 can inhibit proliferation, but not induce apoptosis of B lymphoma cells. Firstly, Mda-7/IL-24 expression was assessed in Raji and Daudi cells by western blotting. The expression of Mda-7/IL-24 protein was weakly detectable in parent cells and cells transfected with the lentiviral vector alone. In contrast, the expression level of Mda-7/IL-24 protein was significantly upregulated in lymphoma cells transfected with Mda-7/IL-24 (Fig. 1A).

MTS assay was performed to investigate the effect of Mda-7/IL-24 on growth of Raji and Daudi cells. As presented in Fig. 1B, Mda-7/IL-24 overexpression significantly inhibited proliferation of lymphoma cells, compared with parent cells and cells transfected with the empty vector.

To further determine the inhibition effect of Mda-7/IL-24 on B lymphoma cells, FCM was performed to analyze whether Mda-7/IL-24 overexpression induced Raji and Daudi cell apoptosis. As presented in Fig. 1C, no difference in the percentage of Annexin V-FITC-positive cells was observed in Raji and Daudi cells overexpressing Mda-7/IL-24, indicating that the growth inhibition observed in these lymphoma cells was not due to induction of cell apoptosis.

The results of FCM also revealed that the percentage of cells in G0/G1 phase was increased, and cells in $\mathrm{S}$ phase were decreased in Raji and Daudi cells overexpressing Mda-7/IL-24, revealing that Mda-7/IL-24 significantly induced the G0/G1 phase arrest in B lymphoma cells (Fig. 1D).

Effect of Mda-7/IL-24 on differentiation of Raji and Daudicells. In order to explore the function of Mda-7/IL-24 in inducing differentiation of B lymphoma cells, FCM was performed to observe the immunophenotype changes of Raji and Daudi cells. It is well known that CD10, CD19, CD45 and CD138 are B lymphocyte differentiation-associated cell surface antigens (14). Therefore, expression of these markers in Raji and Daudi was monitored using FCM. As presented in Fig. 2A, overexpressing Mda-7/IL-24 resulted in significantly decreased expression of the immature B lymphocyte surface marker CD10, and increased expression of the hematopoietic cell mature marker CD45 and the plasma cell marker CD138, but there were no changes in expression of CD19 in Raji and Daudi cells, compared with both non-transfected and empty vector-transfected cells. These data indicated that Raji and Daudi cells overexpressing Mda-7/IL-24 may be induced to terminal differentiation of B lymphocyte.

In addition, overexpression of C-myc and N-myc is considered as a malignant biomarker of various tumors, including lymphoma. The results of western blotting assay also demonstrated that the expression of $\mathrm{C}$-myc and $\mathrm{N}$-myc were all decreased in Raji and Daudi cells overexpressing Mda-7/IL-24 (Fig. 2B), indicating that the malignant phenotype of $\mathrm{B}$ lymphoma cells was mitigated.

In summary, these results revealed that Mda-7/IL-24 has an ability to induce Raji and Daudi cell terminal differentiation. 
A

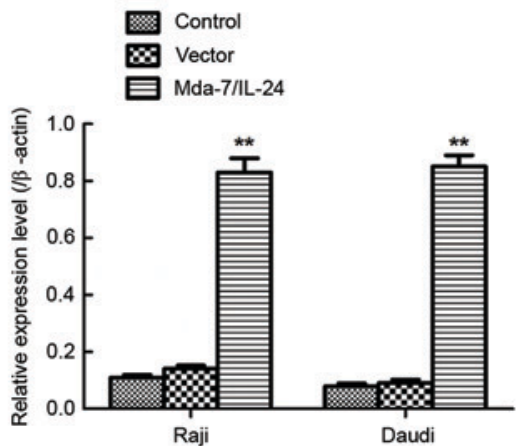

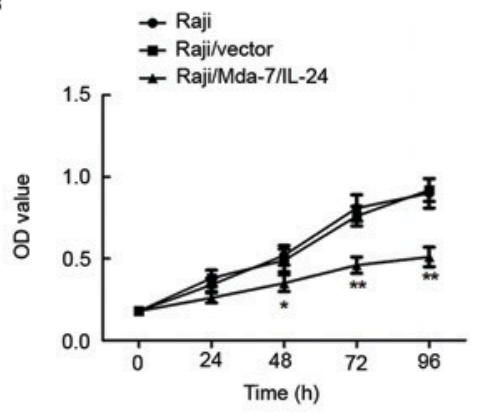
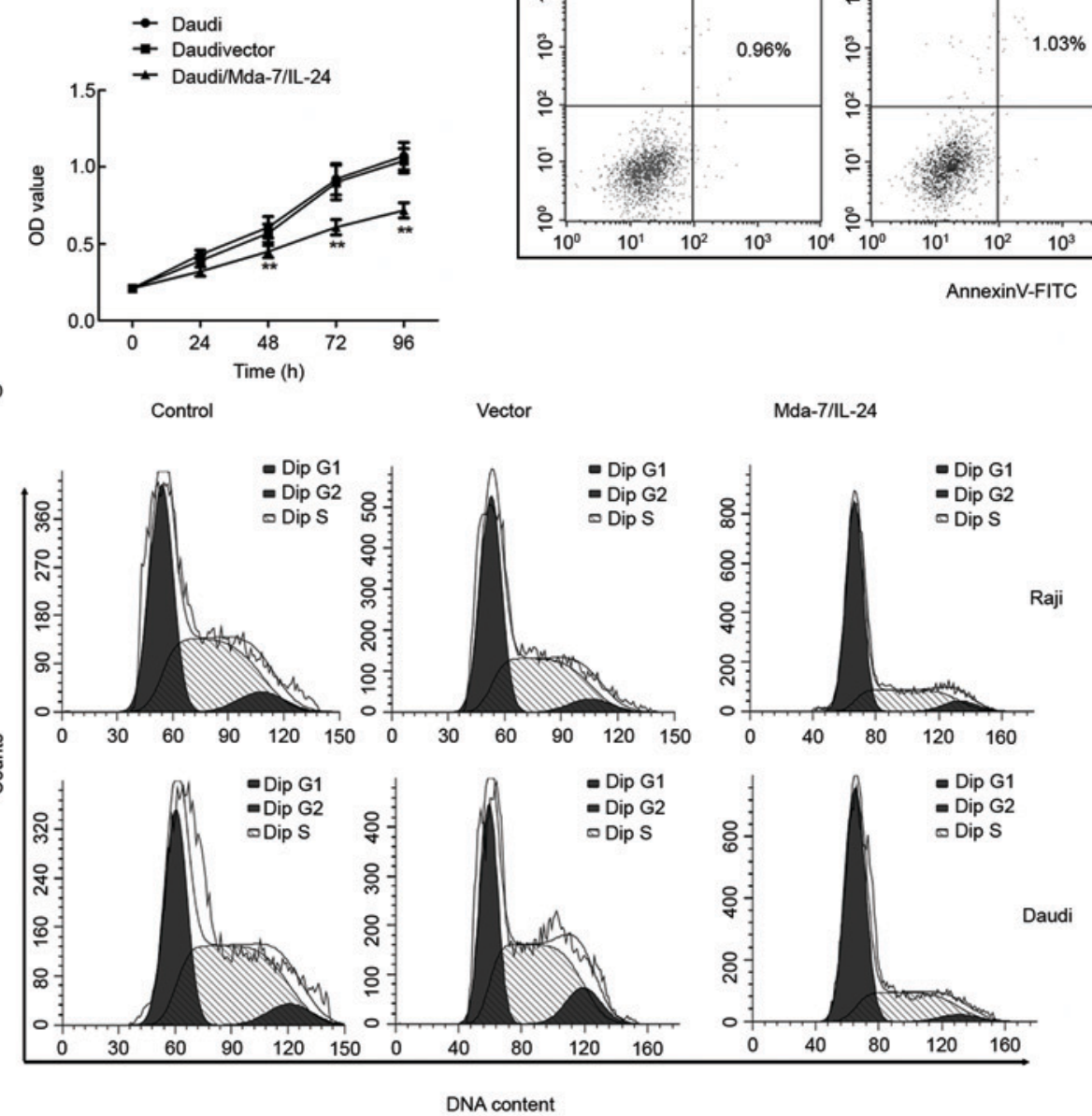

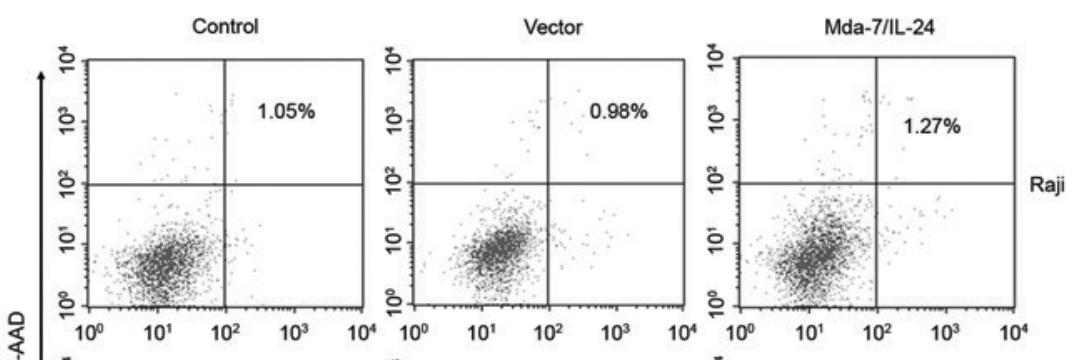

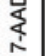

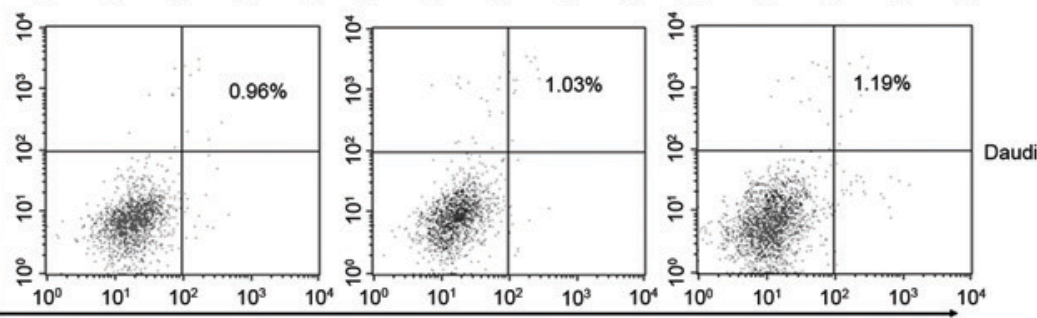

AnnexinV-FITC

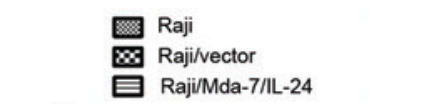

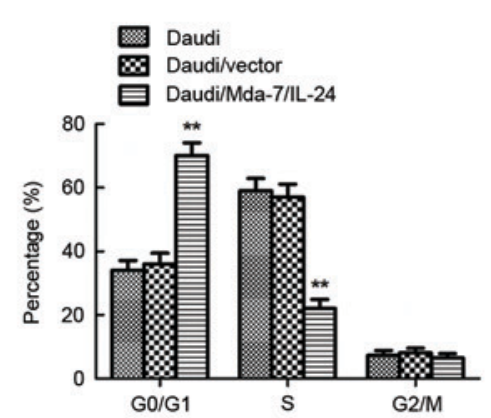

Figure 1. Levels of expression Mda-7/IL-24 in different clones of stably transfected lymphoma cells, and effect of Mda-7/IL-24 on proliferation and apoptosis of Raji and Daudi cells. Control, parent cell lines; cell lines; vector, cells stably transfected with vector alone; Mda-7/IL-24 groups, cells stably transfected with vector expressing Mda-7/IL-24. (A) Mda-7/IL-24 protein expression analyzed by western blotting. $\beta$-actin served as a loading control. (B) Proliferation of cells. (C) Annexin V-FITC staining of cells. (D) Cell cycle analysis, as assessed by flow cytometry. Data are presented as the mean \pm standard deviation of three independent experiments. ${ }^{*} \mathrm{P}<0.05,{ }^{* *} \mathrm{P}<0.01$ vs. control and vector groups. OD, optical density; 7-AAD, 7-aminoactinomycin $\mathrm{D}$; FITC, fluorescein isothiocyanate; Mda-7/IL-24, melanoma differentiation associated gene7/interleukin-24.

Mda-7/IL-24 inhibits migration and invasion of Raji and Daudi cells in vitro. Metastasis is an important malignant feature of lymphoma cells. Migration and invasion assays demonstrated that the numbers of cells migrating into Transwell filters were significantly reduced in Raji and Daudi cells overexpressing Mda-7/IL-24 (Fig. 3A). In addition, the 
A
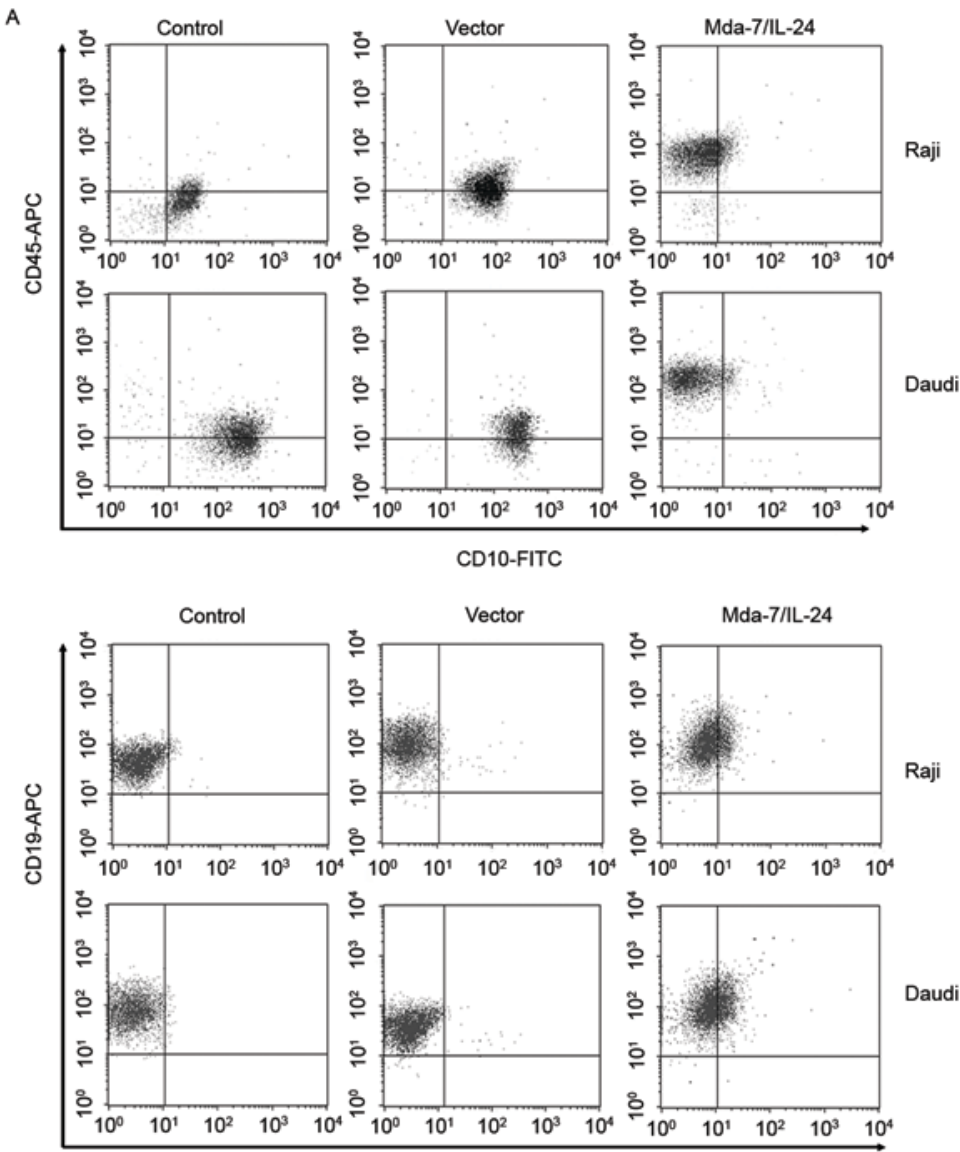

B

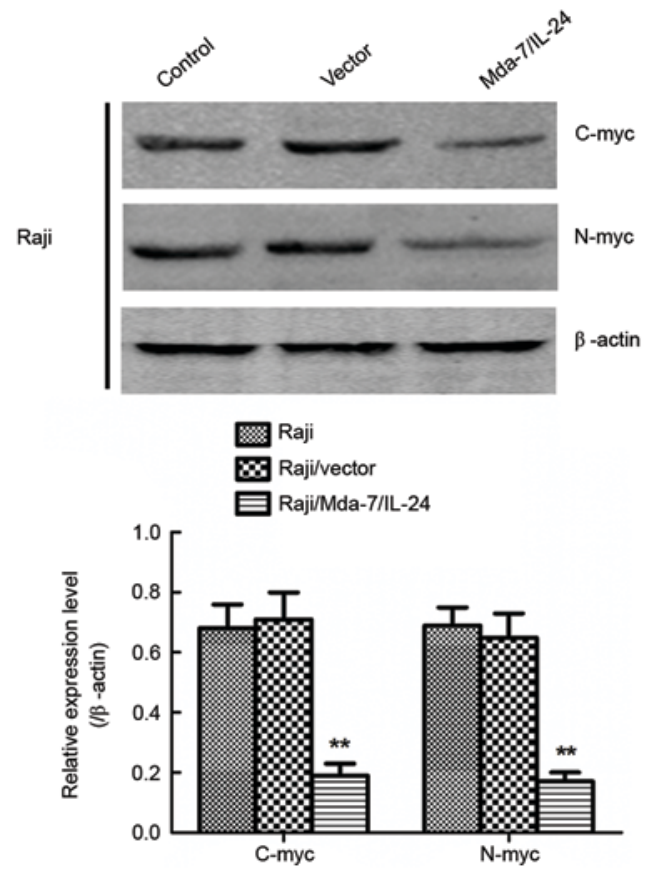

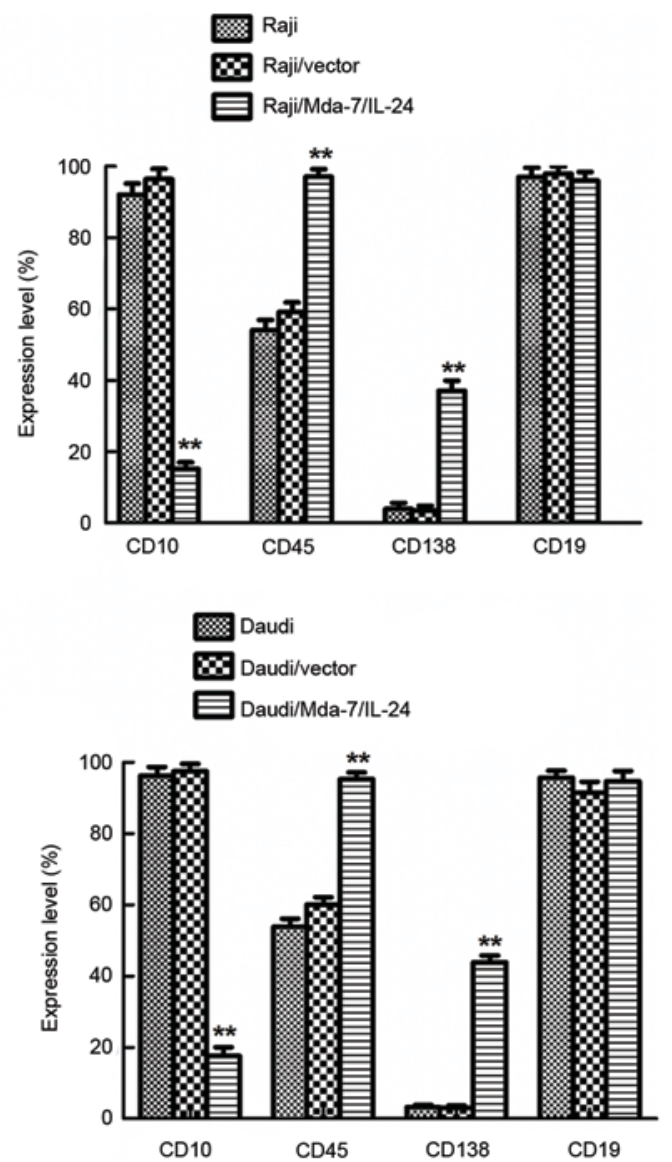
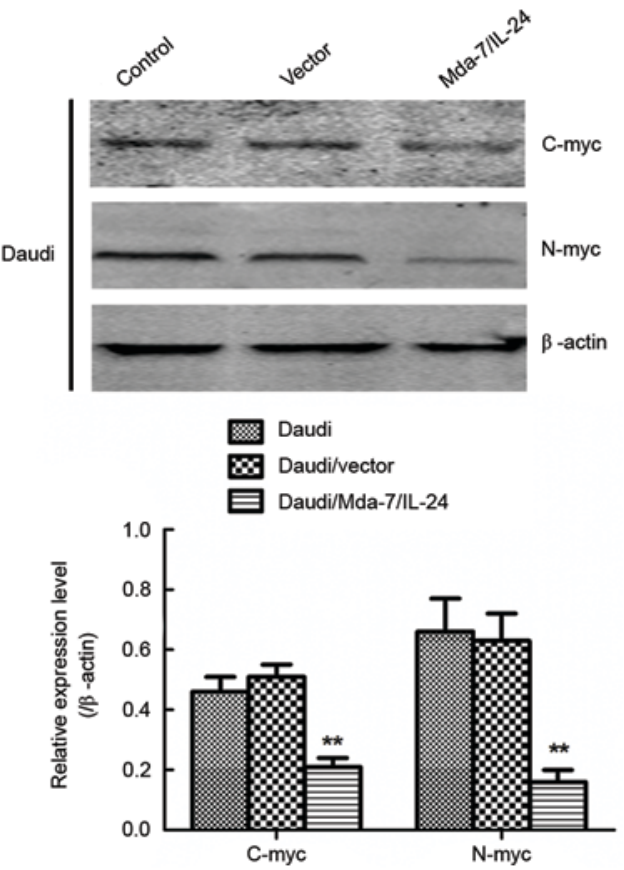

Figure 2. Induction of differentiation by Mda-7/IL-24 in stably transfected lymphoma cells. (A) Effect of Mda-7/IL-24 on the immunophenotype of Raji and Daudi cells, as detected by flow cytometry analysis. (B) Protein expression levels of C-myc and N-myc, two malignant markers of tumors, in Raji and Daudi cells overexpressing Mda-7/IL-24, asdetermined by western blotting. $\beta$-actin served as a loading control. Data are presented as the mean \pm standard deviation of three independent experiments. ${ }^{*} \mathrm{P}<0.05,{ }^{* *} \mathrm{P}<0.01$ vs. control and vector groups. FITC, fluorescein isothiocyanate; Mda-7/IL-24, melanoma differentiation associated gene7/interleukin-24; APC, allophycocyanin; CD, cluster of differentiation.

MMP family serves a key role in metastasis of malignant cells. As expected, MMP2 and MMP9 protein expression levels were decreased in Raji and Daudi cells overexpressing Mda-7/IL-24 (Fig. 3B). 
A

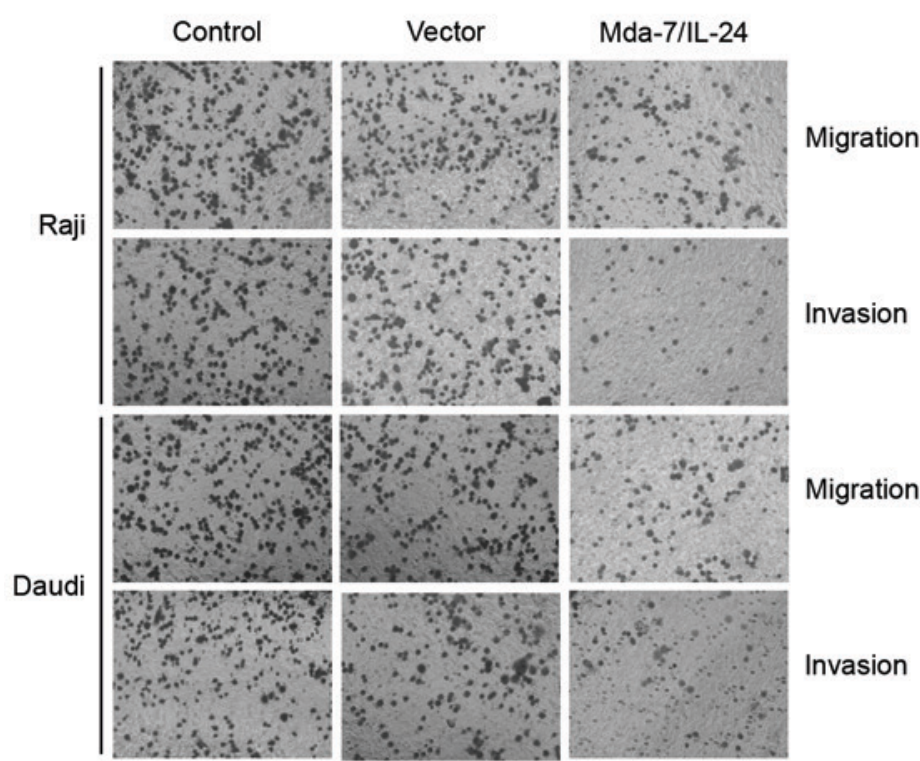

B

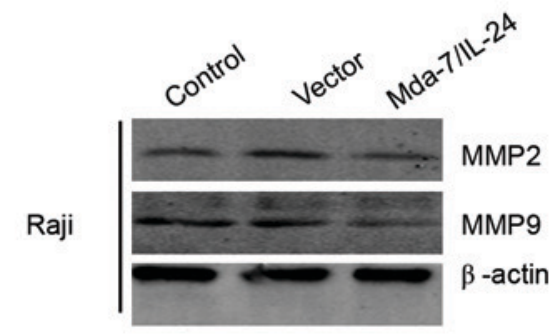

Raji

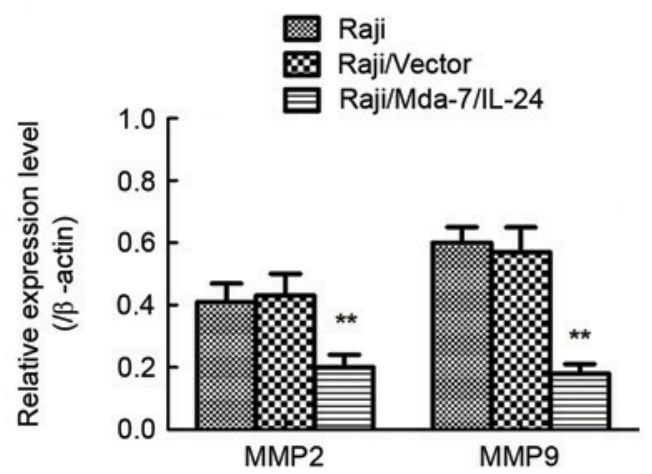

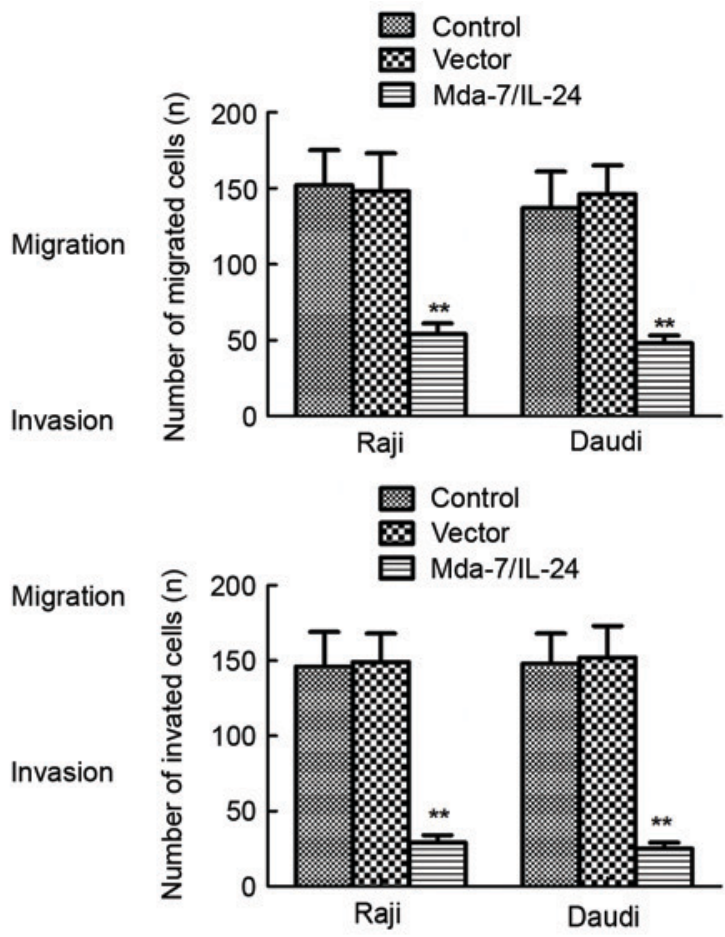
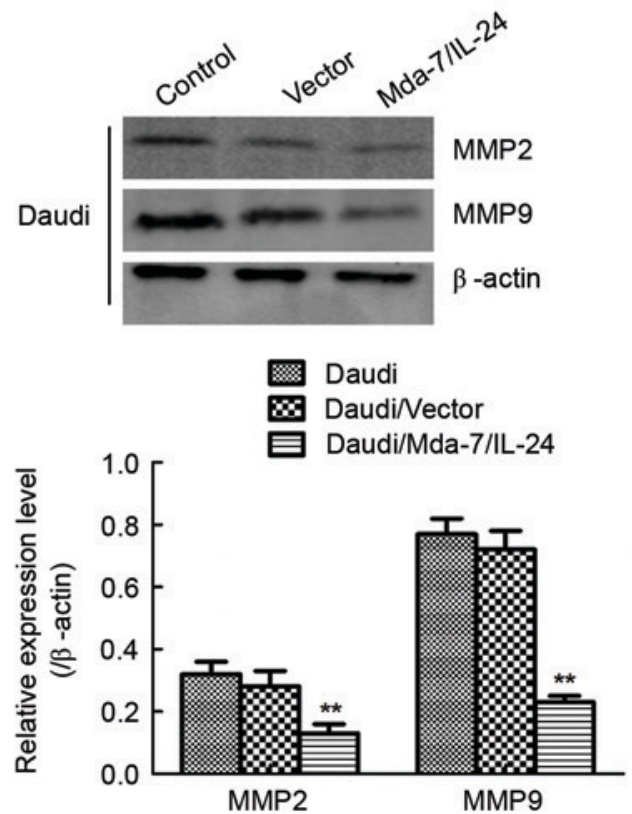

Figure 3. Effects of Mda-7/IL-24 on the migration and invasion ability of Raji and Daudi cells (x200 magnification). (A) Representative Transwell and Matrigel assay images, and quantification of migrated and invaded cells. (B) Western blotting of the protein expression levels of MMP2 and MMP9 in Raji and Daudi cells. $\beta$-actin served as an internal loading control. Data are presented as the mean \pm standard deviation of three independent experiments. ${ }^{* *} \mathrm{P}<0.01$ vs. control and vector groups. Mda-7/IL-24, melanoma differentiation associated gene7/interleukin-24.

Taken together, these results indicated that Mda-7/IL-24 is capable of inhibiting the malignant phenotype of Raji and Daudi cells.

Influence onBlimpl and Bcl6 expression may be involved in Mda-7/IL-24-induced differentiation of Raji and Daudi cells. To determine the underlying mechanism that may account for the Mda-7/IL-24-mediated differentiation-inducing effect in B lymphoma cells, the present study investigated alterations in the expression levels of differentiation-inducing proteins. As Blimp1 and Bcl6 are key proteins associated with the induction of terminal differentiation of B lymphocyte (14), their expression levels were analyzed in Raji and Daudi cells transfected with Mda-7/IL-24. The results revealed that the expression of $\mathrm{Bcl} 6$ protein was decreased, whereas the expression of Blimp1 was increased in the lymphoma cells overexpressing Mda-7/IL-24 (Fig 4). It has been reported that the terminal B cell gene-expression program is induced by loss Bcl6 and by the subsequent increased levels of Blimp1 (15). These results revealed that the differentiation-inducing proteins Blimpl and Bc16 may be involved in the Mda-7/IL-24-mediated differentiation-inducing effect. 

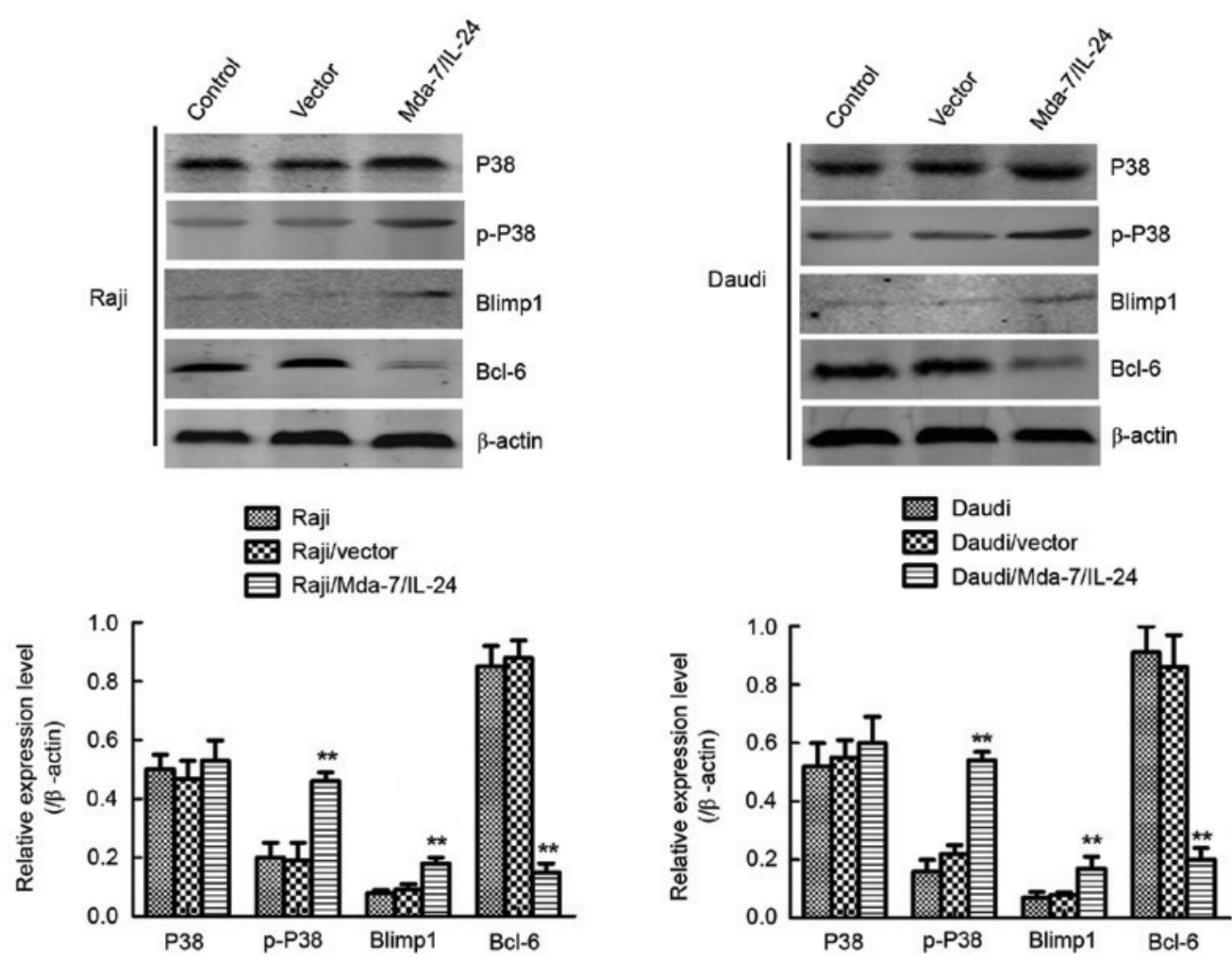

Figure 4. Effects of Mda-7/IL-24 on the expression of proteins associated with differentiation and the P38 mitogen activated protein kinase signaling pathway in Raji and Daudi cells. Western blotting of the protein expression levels of Blimp1, Bcl6, P38 and p-P38 in Raji and Daudi cells. $\beta$-actin served as an internal loading control. Data are presented as the mean \pm standard deviation of three independent experiments. ${ }^{* *} \mathrm{P}<0.01$ vs. control and vector groups. Mda-7/IL-24, melanoma differentiation associated gene7/interleukin-24; Blimp1, PR domain zinc finger protein 1; Bcl6, B-cell lymphoma 6; p, phosphorylated.

To further address the signaling pathway influenced by Mda-7/IL-24, the effect of Mda-7/IL-24 on the activity of the P38 MAPK signaling pathway that is involved in regulating expression of Blimp1 and Bcl6 expression was assessed. As presented in Fig. 4, the expression of p-P38 was increased significantly in Raji and Daudi overexpressing Mda-7/IL-24, suggesting that Mda-7/IL-24 can upregulate the activities of P38 MAPK signaling pathway. Previous reports have suggested that Blimp1 is positively, while Bcl6 is negatively regulated by the P38 signaling pathway (15-17). Therefore, P38 signaling pathway activation may be an important event during lymphoma cell differentiation induced by Mda-7/IL-24.

Furthermore, to elucidate the underling mechanism of the P38 signaling pathway in Mda-7/IL-24-induced cell differentiation, a specific P38 MAPK inhibitor was used to examine proliferation and differentiation of Raji and Daudi cells. Raji and Daudi cells were pre-treated with the specific P38 MAPK inhibitor SB203580 $(20 \mu \mathrm{M})$ for $1 \mathrm{~h}$ before transfection. As presented in Fig. 5A, SB203580 had no significant effect on proliferation of Raji and Daudi cells transfected with the vector alone. However, SB203580 impeded the growth arrest induced by Mda-7/IL-24. Furthermore, inhibition of the P38 MAPK pathway by SB203580 markedly decreased the expression of CD45 and CD138, while increased the expression of CD10 in Raji and Daudi cells overexpressing Mda-7/IL-24 (Fig. 5B). Furthermore, the expression level of C-myc, N-myc and Bcl6 were upregulated, while the expression level of p-P38 and Blimp1 was downregulated significantly by SB203580 in Raji and Daudi cells overexpressing Mda-7/IL-24 (Fig. 6). Collectively, the above results suggested that the activated P38
MAPK signaling pathways induced by Mda-7/IL-24 may be the key factor regulating the terminal differentiation of Raji and Daudi cells.

\section{Discussion}

Previous studies have reported that Mda-7/IL-24 could induce terminal differentiation in human AML cell lines (3), but whether it serves a similar role in lymphoma cells has not been elucidated. The present study revealed that transfection of Mda-7/IL-24 induced mature differentiation of Raji and Daudi lymphoma cells, which were characterized by proliferation inhibition, cell cycle arrest, cell surface antigens changes and decreased expression of malignant markers.

In the present study, the results of the MTS assay demonstrated that transfection with endogenous Mda-7/IL-24 can significantly inhibit growth of Raji and Daudi cells. Additionally, there was no significant increase of apoptosis level in the two lymphoma cells overexpressing Mda-7/IL-24, suggesting that inducing apoptosis may not be involved in the proliferation inhibition of Raji and Daudi cells by Mda-7/IL-24.

The cell cycles of Raji and Daudi cells were arrested in G0/G1 phase. The results of FCM also revealed that overexpression of Mda-7/IL-24 resulted in the significantly decreased expression of CD10, and increased expression of CD45 and CD138, but no changes inexpression of the specific B cell surface antigen CD19 in Raji and Daudi cells. These data revealed that Raji and Daudi cells overexpressing Mda-7/IL-24 may be induced to terminal differentiation of B lymphocytes. In addition, it has been reported that the increased expression 


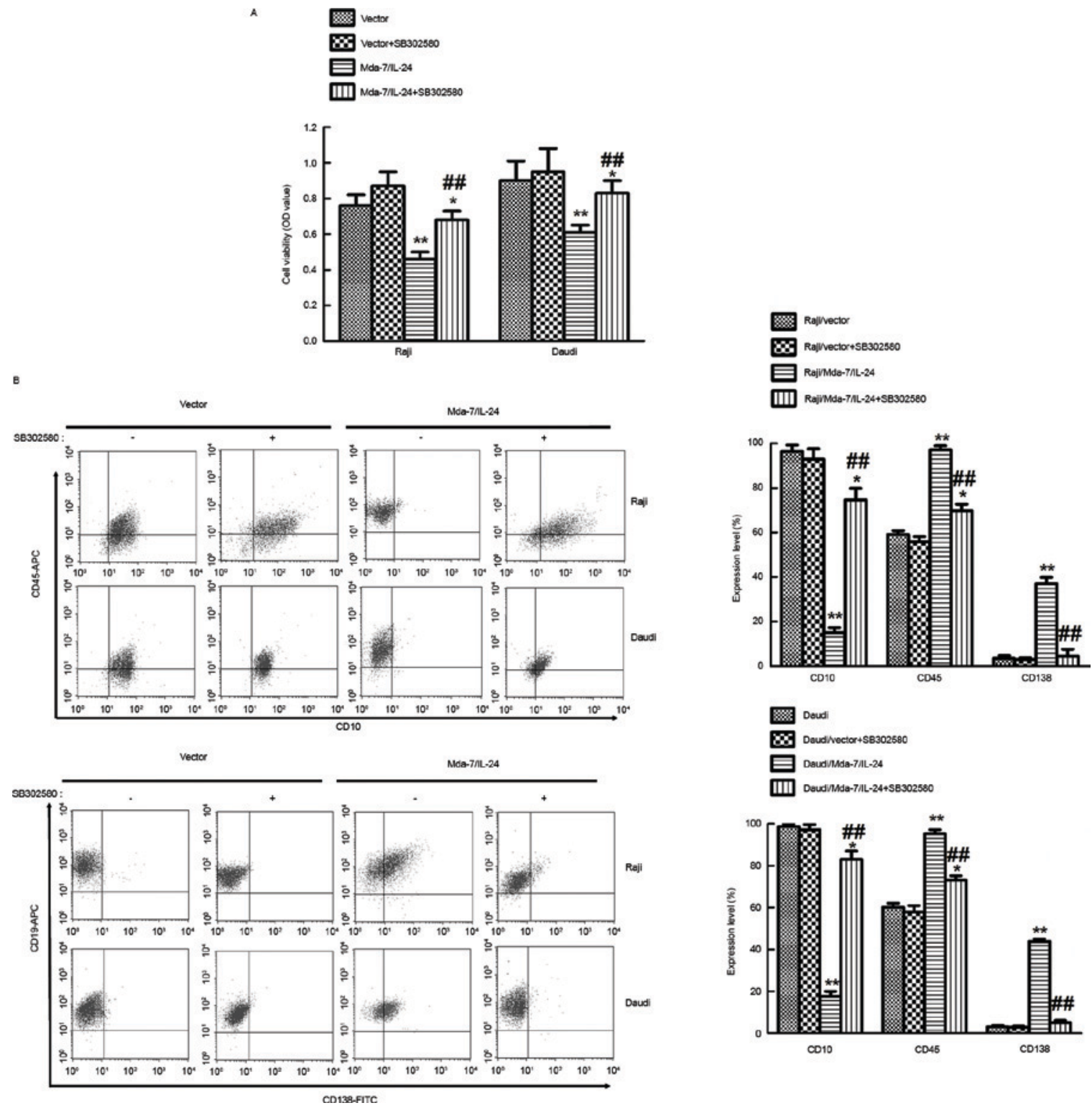

Figure 5. Effects of the P38 mitogen activated protein kinase inhibitor SB302580 on Mda-7/IL-24-mediated proliferation inhibition and differentiation of Raji and Daudi cells. (A) Effect of SB302580 on proliferation inhibition of Raji and Daudi cells induced by Mda-7/IL-24. (B) Effect of SB302580 on the expression of CD10, CD45 and CD138 in Raji and Daudi cells overexpressing Mda-7/IL-24. Data are presented as the mean \pm standard deviation of three independent experiments. " $\mathrm{P}<0.05,{ }^{* *} \mathrm{P}<0.01$ vs. control and vector groups; ${ }^{\# \#} \mathrm{P}<0.01$ vs. Mda-7/IL-24 group. FITC, fluorescein isothiocyanate; Mda-7/IL-24, melanoma differentiation associated gene7/interleukin-24; APC, allophycocyanin; CD, cluster of differentiation; OD, optical density.

of C-myc and N-myc will lead to cell malignant proliferation and neoplastic changes through activation of oncogenic signaling in tumor cells $(18,19)$. Furthermore, the overexpression of $\mathrm{C}$-myc and $\mathrm{N}$-myc is widely observed ina variety of malignancies, including $\mathrm{B}$ cell lymphoma, and much studies have demonstrated that elevated expression of C-myc and $\mathrm{N}$-myc blocks differentiation (18-20). Therefore, C-myc and $\mathrm{N}$-myc can be widely used as malignant biomarkers of cancers. In the present study, the expression levels of $\mathrm{C}$-myc and $\mathrm{N}$-myc were both downregulated significantly in Raji and Daudi cells overexpressing Mda-7/IL-24, suggesting that the malignant phenotype of the two cell lines was mitigated by Mda-7/IL-24 Additionally, the migration and invasion abilities of Raji and Daudi cells overexpressing Mda-7/IL-24 were all decreased, also confirming the relative benign phenotype exhibited in lymphoma cells overexpressing Mda-7/IL-24.
To determine the mechanism that may account for Mda-7/IL-24-mediated differentiation induction effect, the present study analyzed the expression of various differentiation-associated proteins in Raji and Daudi cells transfected with Mda-7/IL-24. Abnormal levels of expression of Blimp1 and $\mathrm{Bcl} 6$ are important in regulating differentiation of $\mathrm{B}$ lymphoma (15). Blimp1 is a master protein regulating the transcriptional network that improves B cell terminal differentiation. Bcl6 functions opposite to Blimp1 in regulating differentiation of B lymphocytes, and it can inhibit and block terminal differentiation of B lymphocytes (20-23). High expression of Bcl6 in B cell lymphoma tissues has been associated with poor differentiation grade $(24,25)$. Thus, Mda-7/IL-24-mediated differentiation induction effect may be associated with upregulation of Blimp1 and downregulation of Bc16 expression in Raji and Daudi cells. 

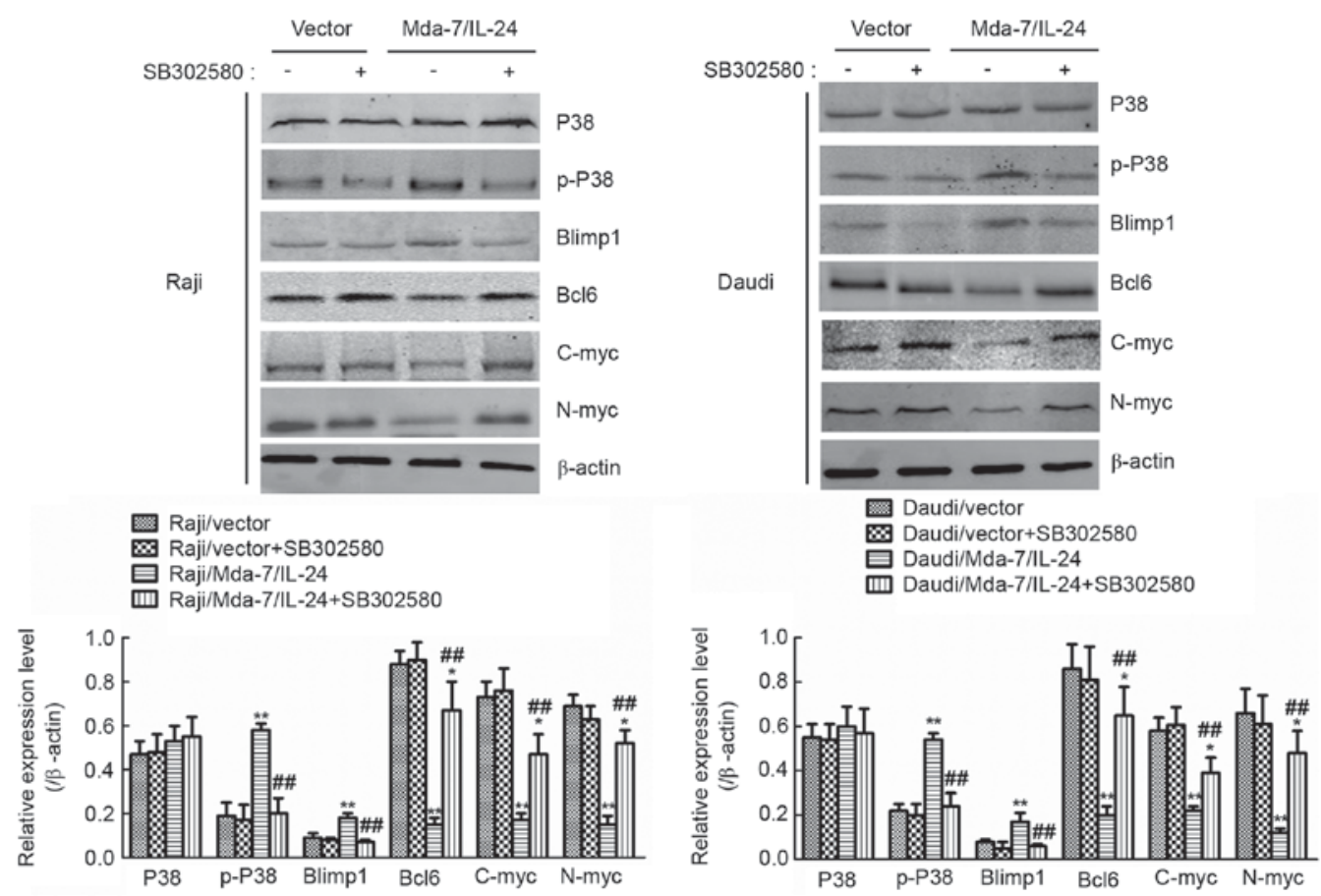

Figure 6. Effects of the P38 mitogen activated protein kinase inhibitor SB302580 on expression of proteins associated with differentiation in Raji and Daudi cells. Western blotting of the protein expression levels of P38, p-P38, Blimp1, Bc16, C-myc and N-myc in Raji and Daudi cells; $\beta$-actin served as an internal loading control. Data are presented as the mean \pm standard deviation of three independent experiments. ${ }^{*} \mathrm{P}<0.05,{ }^{* *} \mathrm{P}<0.01$ vs. control and vector groups; ${ }^{\# \#} \mathrm{P}<0.01$ vs. Mda-7/IL-24 group. Mda-7/IL-24, melanoma differentiation associated gene7/interleukin-24; Blimp1, PR domain zinc finger protein 1; Bcl6, B-cell lymphoma 6; p, phosphorylated.

Furthermore, the present study assessed the activities of the P38 MAPK signaling pathway in Raji and Daudi cells transfected with endogenous Mda-7/IL-24. It is well known that the activity of P38 MAPK pathway serves key role in regulating the expression of Blimp1 and Bcl6 proteins $(20,23)$. A previous study indicated that activation of the P38 MAPK signaling pathway resulted in increased expression of Blimp1 and decreased expression of Bcl6, and that increased activity of the P38 MAPK pathway was detected in various solid tumor cells overexpressing Mda-7/IL-24 (23). In the present study, overexpressing Mda-7/IL-24 also resulted in a similar effecton B lymphoma cells. Additionally, blocking the P38 MAPK pathway using SB203580 markedly impeded Raji and Daudi cell differentiation induced by Mda-7/IL-24. Therefore, Mda-7/IL-24-induced activation of the P38 MAPK signaling pathway in Raji and Daudi cells overexpressing Mda-7/IL-24maybe a key factor in improving the differentiation of B lymphoma cells.

In conclusion, the present study demonstrated that Mda-7/IL-24 can induce mature differentiation of B lymphoma cells by regulating the expression of Blimp1 and Bcl6 via the P38 MAPK signaling pathway. Therefore, Mda-7/IL-24 may be a potential differentiationtherapeutic agent for the clinical treatment of B cell lymphoma.

\section{Acknowledgements}

The present study work was supported by the Natural Science Foundation of China (grant no. 81372200), the Natural Science Foundation of China of Hebei Province (grant no. H2015206376), the Financial Department of Hebei
Province (grant no. 20142065) and the Health Department of Hebei Province (grant no. 20160171).

\section{References}

1. Morin RD, Mendez-Lago M, Mungall AJ, Goya R, Mungall KL, Corbett RD, Johnson NA, Severson TM, Chiu R, Field M, et al: Frequent mutation of histone-modifying genes in non-Hodgkin lymphoma. Nature 476: 298-303, 2011.

2. Ma M, Zhao L, Sun G, Zhang C, Liu L, Du Y, Yang X and Shan B: Mda-7/IL-24 enhances sensitivity of B cell lymphoma to chemotherapy drugs. Oncol Rep 35: 3122-3130, 2016.

3. Yang BX, Duan YJ, Dong CY, Zhang F, Gao WF, Cui XY Lin YM and Ma XT: Novel functions for mda-7/IL-24 and IL-24 delE5: Regulation of differentiation of acute myeloid leukemic cells. Mol Cancer Ther 10: 615-625, 2011.

4. Dong CY, Zhang F, Duan YJ, Yang BX, Lin YM and Ma XT: Mda-7/IL-24 inhibits the proliferation of hematopoietic malignancies in vitro and in vivo. Exp Hematol 36: 938-946, 2008.

5. Sahoo A and Im SH: Molecular Mechanisms Governing IL-24 Gene Expression. Immume Net 12: 1-7, 2012.

6. Huo W, Li ZM, Zhu XM, Bao YM and An LJ: MDA-7/IL-24 suppresses tumor adhesion and invasive potential in hepatocellular carcinoma cell lines. Oncol Rep 30: 986-992, 2013.

7. Rahmani M, Mayo M, Dash R, Sokhi UK, Dmitriev IP, Sarkar D, Dent P, Curiel DT, Fisher PB and Grant S: Melanoma differentiation associated gene-7/interleukin-24 potently induces apoptosis in human myeloid leukemia cells through a Process regulated by endoplasmic reticulum stress. Mol Pharmacol 78: 1096-1104, 2010.

8. Qian W, Liu J, Tong Y, Yan S, Yang C, Yang M and Liu X: Enhanced antitumor activity by a selective conditionally replicating adenovirus combining with MDA-7/interleukin-24 for B-lymphoblastic leukemia via induction of apoptosis. Leukemia 22: 361-369, 2008.

9. Gopalan B, Shanker M, Chada S and Ramesh R: MDA-7/IL-24 suppresses human ovarian carcinoma growth in vitro and in vivo. Mol Cancer 6: 11, 2007. 
10. Menezes ME, Shen XN, Das SK, Emdad L, Guo C, Yuan F, Li YJ Archer MC, Zacksenhaus E, Windle JJ, et al: MDA-7/IL-24 functions as a tumor suppressor gene in vivo in transgenic mouse models of breast cancer. Oncotarget 6: 36928-36942, 2015.

11. Yan F, Bai LP, Gao H, Zhu CM, Lin L and Kang XP: EGF Reverses Multi-drugs Resistance via the p-ERK pathway in HepG2/ADM and SMMC7721/ADM hepatocellular carcinoma models. Asian Pac J Cancer Prev 15: 2619-2623, 2014.

12. Jaffe ES: The 2008 WHO classification of lymphomas: implications for clinical practice and translational research. Hematology Am Soc Hematol Educ Program 523-531, 2009.

13. Zelenetz AD, Gordon LI, Wierda WG, Abramson JS, Advani RH, Andreadis CB, Bartlett N, Byrd JC, Czuczman MS, Fayad LE, Fisher RI, et al: Non-Hodgkin's lymphomas, version 4.2014. J Natl Compr Canc Netw 12: 1282-1303, 2014.

14. Chen S, Wang Z, Dai X, Pan J, Ge J, Han X, Wu Z, Zhou X and Zhao T: Re-expression of microRNA-150 induces EBV-positive Burkitt lymphoma differentiation by modulating c-Myb in vitro. Cancer Sci 104: 826-834, 2013.

15. Lin KI, Kao YY, Kuo HK, Yang WB, Chou A, Lin HH, Yu AL and Wong CH: Reishi Polysaccharides induce immunoglobulin production through the TLR4/TLR2-mediated induction of transcription factor blimp-1. J Biol Chem 281: 24111-24123, 2006.

16. Xu S, Oshima T, Imada T, Masuda M, Debnath B, Grande F, Garofalo A and Neamati N: Stabilization of MDA-7/IL-24 for colon cancer therapy. Cancer Lett 335: 421-430, 2013.

17. Patani N, Douglas-Jones A, Mansel R, Jiang W and Mokbel K: Tumor suppressor function of MDA-7/IL-24 in human breast cancer. Cancer Cell Int 10: 29, 2010.

18. Han B, Li W, Sun Y, Zhou L, Xu Y and Zhao X: A prolyl-hydroxylase inhibitor, ethyl-3,4-dihydroxybenzoate, induces cell autophagy and apoptosis in esophageal squamous cell carcinoma cells via up-regulation of bnip3 and n-myc downstream-regulated gene-1. PLoS One 9: e107204, 2014.
19. Wei W, Bracher-Manecke JC, Zhao X, Davies NH, Zhou L, Ai R, Oliver L, Vallette F and Hendricks DT: Oncogenic but non-essential role of $\mathrm{N}$-myc downstream regulated gene 1 in the progression of esophageal squamous cell carcinoma. Cancer Biol Ther 14: 164-174, 2013.

20. Chan YH, Chiang MF, Tsai YC, Su ST, Chen MH, Hou MS and Lin KI: Absence of the transcriptional repressor blimp-1 in hematopoietic lineages reveals its role in dendritic cell homeostatic development and function. J Immunol 183: 7039-7046, 2009.

21. Park YS, Kang JW, Lee DH, Kim MS, Bak Y, Yang Y, Lee HG, Hong J and Yoon DY: Interleukin-32 $\alpha$ downregulates the activity of the B-cell CLL/lymphoma 6 protein by inhibiting protein kinase Ce-dependent SUMO-2 modification. Oncotarget 5: 8765-8777, 2014

22. Zheng T, Chen L, Noh AL and Yim M: Cetylpyridinium chloride inhibits receptor activator of nuclear factor- $\kappa \mathrm{B}$ ligand-induced osteoclast formation. Biol Pharm Bull 36: 509-514, 2013.

23. Altieri P, Spallarossa P, Barisione C, Garibaldi S, Garuti A, Fabbi P, Ghigliotti $\mathrm{G}$ and Brunelli $\mathrm{C}$ : Inhibition of doxorubicin-Induced senescence by PPAR $\delta$ activation agonists in cardiac muscle cells: Cooperation between PPARd and Bcl6. PLoS One 7: e46126, 2012

24. Ott G, Rosenwald A and Campo E: Understanding MYC-driven aggressive B-celll ymphomas: Pathogenesis and classification. Blood 122: 3884-3891, 2013.

25. Randen U, Trøen G, Tierens A, Steen C, Warsame A, Beiske K, Tjønnfjord GE, Berentsen S and Delabie J: Primary cold agglutinin-associated lymphoproliferative disease: a B-cell lymphoma of the bone marrow distinct from lymphoplasmacytic lymphoma. Haematologica 99: 497-504, 2014. 